\title{
Serum Leptin is not Altered nor Related to Cognitive Decline in Alzheimer's Disease
}

\author{
Charlotte E. Teunissen ${ }^{\mathrm{a}, *}$, Wiesje M. van der Flier ${ }^{\mathrm{b}, \mathrm{c}}$, Philip Scheltens ${ }^{\mathrm{b}}$, Anneli Duits ${ }^{\mathrm{a}}$, \\ Nienke Wijnstok $^{\mathrm{d}, \mathrm{e}}$, Giel Nijpels ${ }^{\mathrm{e}, \mathrm{f}}$, Jacqueline M. Dekker ${ }^{\mathrm{c}, \mathrm{e}}$, M.A. Blankenstein ${ }^{\mathrm{a}}$ \\ and Annemieke C. Heijboer ${ }^{\mathrm{a}}$ \\ ${ }^{a}$ Department of Clinical Chemistry, Neuroscience Campus Amsterdam, VU University Medical Center, Amsterdam, \\ the Netherlands \\ ${ }^{\mathrm{b}}$ Alzheimer center \& Department of Neurology, Neuroscience Campus Amsterdam, VU University Medical Center, \\ Amsterdam, the Netherlands \\ ${ }^{\mathrm{c}}$ Department of Epidemiology and Biostatistics, VU University Medical Center, Amsterdam, the Netherlands \\ ${ }^{\mathrm{d}}$ Department of Health Sciences, Faculty of Earth and Life Sciences, VU University, Amsterdam, the Netherlands \\ ${ }^{\mathrm{e}}$ EMGO + Institute for Health and Care Research, VU University Medical Center, Amsterdam, the Netherlands \\ ${ }^{\mathrm{f}}$ Department of General Practice, VU University Medical Center, Amsterdam, the Netherlands
}

Accepted 23 September 2014

\begin{abstract}
.
Background: Low plasma leptin levels can be a risk factor for Alzheimer's disease (AD) but the relation of leptin with disease progression in clinical AD is unknown.

Objective: The aim of this study was to investigate the relation between serum leptin concentrations and cognitive decline in clinical AD.

Methods: Serum leptin levels were analyzed in 295 non-obese subjects including healthy controls $(n=65)$, patients with subjective memory complaints $(n=99)$, patients with $\mathrm{AD}(n=100)$, and patients with vascular dementia $(n=31)$. Leptin levels were related to hippocampal atrophy, baseline Mini-Mental State Examination (MMSE) scores and annual decline in MMSE measured over 2 years (range $0.4-4.5$ years).

Results: Serum leptin levels were similar in AD patients compared to healthy controls and patients with subjective memory complaints. No correlation was observed between leptin concentrations and MMSE, annual change in MMSE during follow-up or atrophy.

Conclusion: Serum leptin levels are not altered in this population of relatively young AD or vascular dementia patients (mean 60) compared to healthy and clinical control groups and were not related to cognitive decline. These results suggest that peripheral leptin levels do not play a role in evolution of AD pathology.
\end{abstract}

Keywords: Alzheimer's disease, atrophy, blood-based biomarker, cognition, leptin, vascular dementia

\section{INTRODUCTION}

Leptin is an endocrine regulator of satiety and food intake, can promote weight loss, and is predictive for long-term weight gain. In vitro and animal studies

\footnotetext{
*Correspondence to: Charlotte Teunissen, Neurochemistry Laboratory and Biobank, Department of Clinical Chemistry, VU University Medical Center, Amsterdam, PO Box 7057, 1007 MB Amsterdam, the Netherlands. Tel: +31 20 4443680; Fax: +31 20 4443895; E-mail: c.teunissen@vumc.nl.
}

indicate a role for leptin in cognition and AD pathology [1]. Moreover, leptin supplementation to deficient persons leads to improved cognitive function [2]. Therefore, a relation between $\mathrm{AD}$ development and leptin is suspected. In two longitudinal follow-up studies, high plasma leptin levels appeared to decrease the risk of AD [3, 4]. In the first study, 785 communitydwelling non-demented persons from the Framingham study (mean age 79.9 years) were followed for up to 8 years. Higher leptin levels were associated with lower 
risk on incident $\mathrm{AD}$ (hazard ratio [HR] 0.60 for $\mathrm{AD}$ ) [3]. In the second study, 2,019 Chinese non-demented persons (mean age 72-75 years) were followed for 5 years and higher baseline leptin levels were weakly, though significantly, associated with decreased risk of AD development (HR 0.92) [4].

However, this risk reduction associated with high leptin levels is in contrast to the effect of mid-life obesity on $\mathrm{AD}$ [5], as obesity is associated with high leptin levels. It may be that the relation of leptin with AD is different in obese individuals, who show leptin resistance, from non-obese patients. Weight loss (and thus lowering of leptin levels) is a feature of early stages of AD [6]. However, no relation between low body mass index (BMI) and plasma leptin levels was reported in $\mathrm{AD}$ in a recent small study [7]. Previous studies included small patient groups $(n<60)$ and no longitudinal clinical follow-up data of AD patients. The aim of the current study was therefore to investigate the relation of leptin with prevalence and clinical and radiologically assessed severity $\mathrm{AD}$ in a large, welldefined patient cohort of non-obese AD patients. We additionally assessed the relation of leptin with cognitive decline at follow-up. We included two control groups: 1) community healthy controls, and 2) patients with subjective memory complaints (SMC), which is a relevant control group in outpatient settings.

\section{METHODS}

In a cross-sectional study design, a total of 295 subjects were included in this study. Serum from AD patients $(n=100)$, patients with SMC $(n=99)$, and patients with vascular dementia $(\mathrm{VaD})(n=31)$ was selected from the Biobank of the Alzheimer Center Memory Cohort. Venipuncture was performed during diagnostic work-up (no fasting requirement) and blood was spun within $2 \mathrm{~h}(1,800 \mathrm{~g}, 10 \mathrm{~min})$ and immediately stored at $-80^{\circ} \mathrm{C}$ according to consensus guidelines [8]. All patients underwent a standardized dementia screening, including physical and neurological examination as well as laboratory tests, EEG, and brain MRI as described [9]. MMSE was scored at baseline and after an average follow-up of 2 years (Table 1) [10]. Patients were labeled as having subjective complaints when they presented with cognitive complaints, but cognitive and laboratory investigations were normal and criteria for mild cognitive impairment, dementia or any other neurological or psychiatric disorders known to cause cognitive complaints were not met (i.e., cognitively normal elderly). In addition, we included a group of non-obese healthy controls, consisting of an age-matched subset of the New Hoorn study, which is a random sample of all residents aged 40-65 years from the Dutch city of Hoorn who were invited to participate in a study (2006-2007) on glucose metabolism [11]. The study was performed according to the ethical

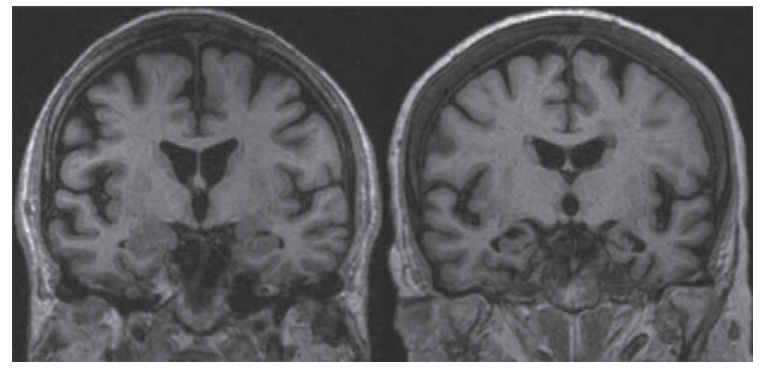

Fig. 1. Representative picture to illustrate the rating of atrophy of the medial temporal lobe (MTA). Atrophy of the MTA was visually rated on coronal reconstructions of 3D T1-weighted MRI, based on the width of the choroid fissure and temporal horn and on the height of the hippocampal formation. A score of 0 indicates absence of atrophy (example: left panel). A score of 4 indicates severe atrophy (right panel: $\mathrm{MTA}=3$ ).

Table 1

Clinical, demographic, and leptin data of the patient population

\begin{tabular}{|c|c|c|c|c|}
\hline & Controls & SMC & $\mathrm{AD}$ & $\mathrm{VaD}$ \\
\hline Characteristics & $n=65$ & $n=100$ & $n=98$ & $n=30$ \\
\hline Age-Years (SD) & $58(7)^{\mathrm{a}, \mathrm{b}, \mathrm{c}}$ & $62(7)^{\mathrm{c}}$ & $63(6)^{\mathrm{c}}$ & $67(8)$ \\
\hline Gender: number of females $(\%)$ & $26(39)$ & $42(42)$ & $47(48)$ & $9(29)$ \\
\hline ApoE4-positivity (\%) & & $39(42.4)$ & $67(72)$ & $12(48)$ \\
\hline $\mathrm{BMI}(\mathrm{SD})$ & $25(2)^{b}$ & $25(3)^{b, c}$ & $24(3)^{c}$ & $27(4)$ \\
\hline MMSE (SD) & - & $28(2)^{\mathrm{b}, \mathrm{c}}$ & $19(5)^{\mathrm{c}}$ & $24(4)$ \\
\hline Annual change in MMSE $(\mathrm{SD})^{\mathrm{d}}$ & & $-0.1(1.1)$ & $-2.6(3.3)$ & $-0.7(2.5)$ \\
\hline Duration clinical follow-up (SD) ${ }^{\mathrm{d}}$ & & $2.1(1.2)$ & $2.1(0.9)$ & $1.9(1.0)$ \\
\hline Medial temporal lobe atrophy score (median (IQR)) [12] & - & $0.0(0.0,0.5)^{\mathrm{b}, \mathrm{c}}$ & $1.0(0.5,2.0)$ & $1.5(1.0,2.0)$ \\
\hline Leptin concentration (median in $\mu \mathrm{g} / \mathrm{L}(\mathrm{IQR})$ ) & $6.3(3.5,13.5)$ & $5.9(3.6,10.4)$ & $5.4(3.3,11.5)$ & $8.2(6.1,16.2)$ \\
\hline
\end{tabular}

SMC, subjective memory complaints; AD, Alzheimer's disease; VaD, vascular dementia; BMI, body mass index; MMSE, Mini-Mental State Exam; IQR, interquartile range; SD, standard deviation. ${ }^{\mathrm{a}}$ significant difference $(p<0.05)$ compared to SMC ${ }^{\mathrm{b}}$ significant difference $(p<0.05)$ compared to $\mathrm{AD}^{\mathrm{c}}$ significant difference $(p<0.05)$ compared to $\mathrm{VD}^{\mathrm{d}} n=135(52 \mathrm{SMC}, 70 \mathrm{AD}, 13 \mathrm{VaD})$. 
principles of the Declaration of Helsinki and was approved by the local ethics committee. We obtained written informed consent from all subjects participating in the study.

Controls (healthy and SMC) and AD patients with a $\mathrm{BMI}>30$, a confirmed diagnosis of diabetes mellitus type II, or a history of diagnosed depression were excluded. All patients using Alzheimer medication (Rivastigmin (Exelon), Galantamin (Reminyl) or Memantin (Ebixa)) were excluded. ApoE genotyping was performed after DNA isolation from $10 \mathrm{~mL}$ EDTA blood, using the Light-Cycler APOE mutation detection method (Roche Diagnostics, GmbH, Mannheim, Germany). Subjects were classified as ApoEع4 negative or positive. Atrophy of the medial temporal lobes was visually rated on a 5-point scale (0-4) [12]. A representative picture is provided in Fig. 1.

Leptin was measured using a radioimmunoassay (Millipore, St Charles, Missouri, USA). The lower limit of quantitation is $0.5 \mu \mathrm{g} / \mathrm{L}$. The inter-assay variation of this assay is $<6 \%$ for the whole concentration range.

Data are presented as means with standard deviation (SD) if normally distributed, and alternatively as medians with interquartile range (IQR). Differences between the groups were calculated using ANOVA of log-transformed leptin values, including BMI, age and gender as covariates and with Scheffe's posthoc tests. Annual change in MMSE was calculated by dividing the difference between MMSE and baseline and follow-up by follow-up duration. Regression analyses were performed including annual change in MMSE as dependent variable, log-transformed leptin values as independent variable, with or without gender, age and BMI as covariates. Correlations were calculated using Pearson correlation coefficients of the log-transformed leptin values. A p-value $<0.05$ was considered as significant.

\section{RESULTS}

Figure 2 shows the serum leptin levels in the different clinical groups included in this study and raw data are presented in Table 1. The results show no difference between healthy controls, subjects with SMC and patients with $\mathrm{AD}$. Leptin levels were two times higher in females than in males $(11.2 \operatorname{IQR}(5.8,19.1)$ in females versus $4.9(3.3,7.2) \mu \mathrm{g} / \mathrm{L}$ in males). Inclusion of gender as covariate in the ANOVA did not change the results, which is also illustrated in Fig. $2 \mathrm{~B}$ and $\mathrm{C}$ showing the leptin levels in each gender separately. Leptin levels were higher in patients with $\mathrm{VaD}$ compared to
SMC and AD patients $(p<0.05)$, but this disappeared after correction for BMI (data not shown).

BMI was correlated to leptin levels in the total group (D), as well as in every separate clinical group ( $\mathrm{r}$ between 0.23 (SMC) and 0.54 in VaD patients).

There was no correlation between leptin levels and age $(r-0.44, p=0.449)$, gender, time of the day of blood withdrawal $(r=0.038, p=0.522)$, medial temporal lobe atrophy $(r=0.009, p=0.890)$, atrophy $(-0.002, p=0.971)$, or number of ApoE4 alleles $(r=0.009, r=0.896)$.

Leptin did not correlate with baseline MMSE in the $\mathrm{AD}$ patients. There was neither a relation of baseline leptin with annual change in MMSE during followup of two years (Fig. 2E). The standardized beta $0.048, p=0.582$ in the uncorrected model and -0.02 , $p=0.872$ in the model corrected for BMI, gender, age).

\section{DISCUSSION}

The results of the current study show stable leptin levels in $\mathrm{AD}$, which did not predict cognitive decline. The stable levels in AD are in line with results of recent studies in clinical AD [7, 13-15]. However, the results seem contradictory to the decrease recently reported in 60 AD compared to 60 controls [16] and results from animal studies suggesting a role of leptin in memory decline and AD pathology, e.g., showing that treatment of CRND8 mice with leptin led to a reduction of $A \beta$ levels and memory improvement [1]. Importantly, the results are in contrast to the finding of large population studies showing that low leptin is a risk factor for $\mathrm{AD}$ $[3,4]$. Thus, while previous results are in line with a role of leptin in $\mathrm{AD}$ development, our results show that altered leptin metabolism as reflected in peripheral blood, is at least not associated with end stage, clinical AD.

The large patient sample and the fact that the expected correlation of leptin with BMI was present give confidence to our current data. Moreover, we also included non-obese persons only, which was similar to the populations in the studies of Zhou and Lieb [3, 4]. Thus, alternative explanations must be sought for the lack of changes in leptin levels in relation to cognitive decline of AD. The difference in disease severity may be the explanation for the discrepant results between the only study that showed decreased serum leptin levels in $\mathrm{AD}$, since they included $\mathrm{AD}$ patients of similar age, but with lower MMSE (10 SD 4) than our group (19 SD 5), and showed a negative correlation between leptin and MMSE [16]. However, the decrease 

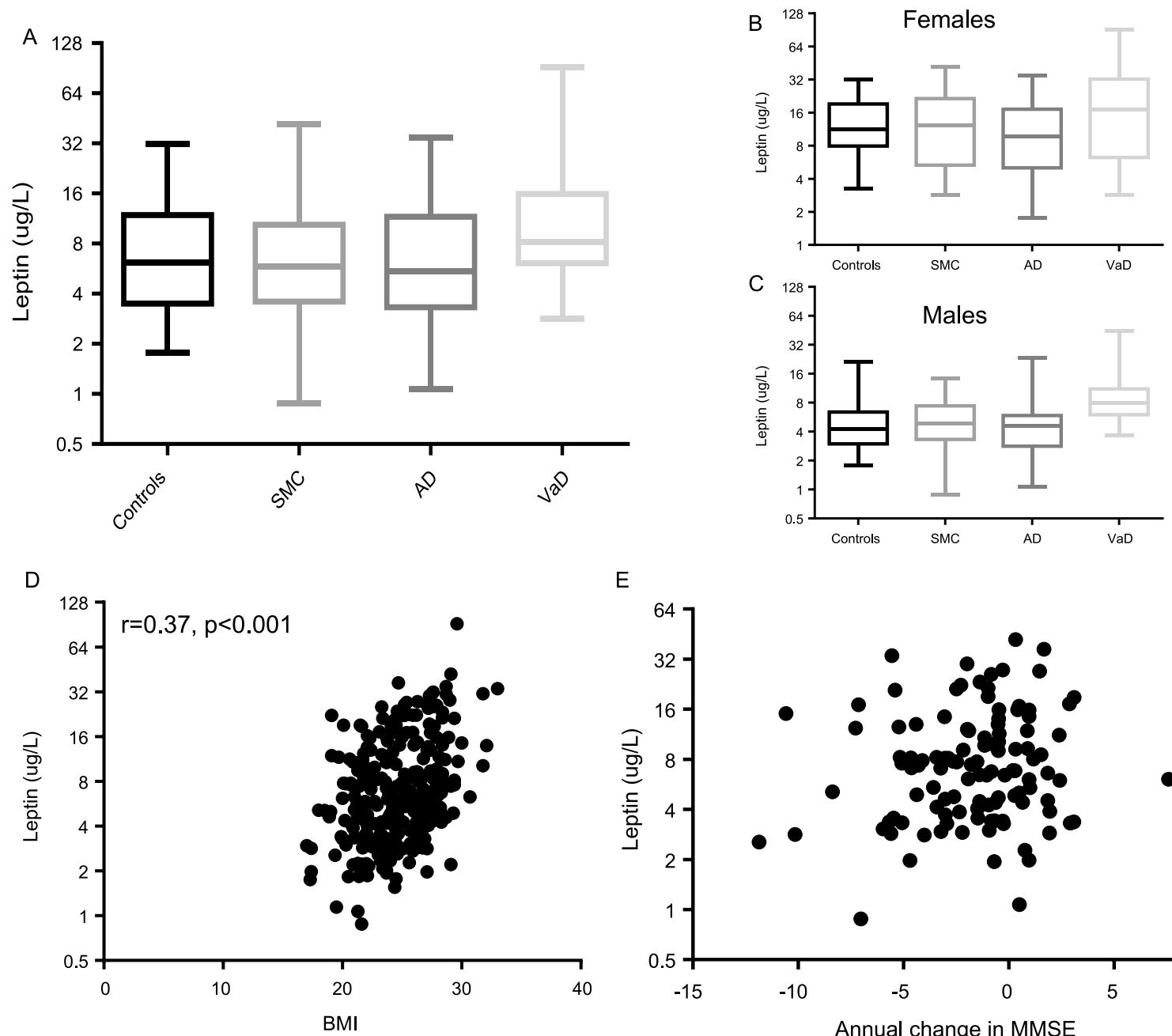

E

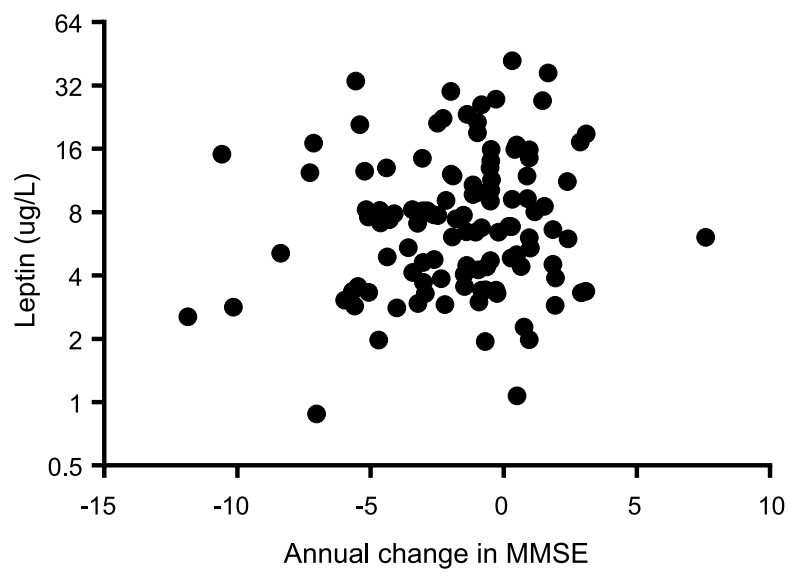

Fig. 2. Serum leptin levels in relation to clinical variables. A) Serum leptin levels were similar in control, SMC and AD patients. The increased level in $\mathrm{VaD}$ disappeared after correction for BMI. Similar patterns were obtained when females (B) and males (C) were analyzed separately. D) Serum leptin correlated positively to BMI values. E) Serum leptin levels were not correlated to annual change in MMSE.

in more severe stages of AD only is incongruent with the reported role of low leptin as a risk factor for $\mathrm{AD}$ $[3,4]$, which implies that it would be decreased in early stages of the disease as well.

One explanation could be that age is an important confounder in the relation between leptin and AD. Aging is the strongest risk factor for getting AD and leptin receptor resistance is related to aging. However, no correlation between leptin and age was present in our study, ruling out a confounding role of aging. Alternatively, aging per se could mask effects of leptin, as other vascular risk factors were shown to be predictive for late-life cognitive functioning specifically after 15 years of follow-up, while the effects became smaller when people grew older [17].
Another explanation could be that the relation between leptin and AD or cognitive decline is influenced by many biological factors, which can be different in the preclinical compared to the clinical stage of $\mathrm{AD}$. $\mathrm{AD}$ has a long preclinical stage and the pathology can start already 10-20 years before clinical visible disease [18]. For example, amyloid and tau biomarkers likely reach their plateau before clinical symptoms as cognitive decline appear [18]. Thus, effects of leptin could be important in the preclinical stage of initial disease development, but may have no further effects at the clinical stage of the disease. Data from an extensive immunohistochemistry study focusing on leptin resistance in $\mathrm{AD}$ tissue and in postmortem cerebrospinal fluid (CSF) support the existence of 
bimodal and region-specific relations [19]. Although numbers of patients included in the CSF analysis in that study were too low to draw strong conclusions, an early stage decrease was observed in CSF of mild cognitive impairment patients and an increase in late Braak stages. The results did, however, not show a correlation between CSF leptin and BMI, which suggests that leptin concentrations and consequent effects are compartmentalized and support that blood levels of leptin do not reflect central nervous system pathology.

In conclusion, cumulative data from our study and the majority of previous studies indicate that blood leptin levels are not substantially changed in clinical AD, and thus it is too early to consider leptin supplementation to $\mathrm{AD}$ patients.

\section{DISCLOSURE STATEMENT}

Authors' disclosures available online (http://www.jalz.com/disclosures/view.php?id=2568).

\section{REFERENCES}

[1] Greco SJ, Bryan KJ, Sarkar S, Zhu X, Smith MA, Ashford JW, Johnston JM, Tezapsidis N, Casadesus G (2010) Leptin reduces pathology and improves memory in a transgenic mouse model of Alzheimer's disease. J Alzheimers Dis 19, 1155-1167.

[2] Paz-Filho G, Wong ML, Licinio J (2010) Leptin levels and Alzheimer disease. JAMA 303, 1478-1479.

[3] Lieb W, Beiser AS, Vasan RS, Tan ZS, Au R, Harris TB, Roubenoff R, Auerbach S, DeCarli C, Wolf PA, Seshadri S (2009) Association of plasma leptin levels with incident Alzheimer disease and MRI measures of brain aging. JAMA 302, 2565-2572.

[4] Zhou R, Deng J, Zhang M, Zhou HD, Wang YJ (2011) Association between bone mineral density and the risk of Alzheimer's disease. J Alzheimers Dis 24, 101-108.

[5] Kivipelto M, Ngandu T, Fratiglioni L, Viitanen M, Kareholt I, Winblad B, Helkala EL, Tuomilehto J, Soininen H, Nissinen A (2005) Obesity and vascular risk factors at midlife and the risk of dementia and Alzheimer disease. Arch Neurol 62, 15561560.

[6] Inelmen EM, Sergi G, Coin A, Girardi A, Manzato E (2010) An open-ended question: Alzheimer's disease and involuntary weight loss: Which comes first? Aging Clin Exp Res 22, 192197.

[7] Theodoropoulou A, Metallinos IC, Psyrogiannis A, Vagenakis GA, Kyriazopoulou V (2012) Ghrelin and leptin secretion in patients with moderate Alzheimer's disease. J Nutr Health Aging 16, 472-477.
[8] Teunissen CE, Tumani H, Engelborghs S, Mollenhauer B (2014) Biobanking of CSF: International standardization to optimize biomarker development. Clin Biochem 47, 288-292.

[9] van der Flier WM, Pijnenburg YA, Prins N, Lemstra AW, Bouwman FH, Teunissen CE, van Berckel BN, Stam CJ, Barkhof F, Visser PJ, van EE, Scheltens P (2014) Optimizing patient care and research: The Amsterdam Dementia Cohort. J Alzheimers Dis 41, 313-327.

[10] Folstein MF, Folstein SE, McHugh PR (1975) "Mini-mental state". A practical method for grading the cognitive state of patients for the clinician. J Psychiatr Res 12, 189-198.

[11] van 't RE, Alssema M, Rijkelijkhuizen JM, Kostense PJ, Nijpels G, Dekker JM (2010) Relationship between A1C and glucose levels in the general Dutch population: The new Hoorn study. Diabetes Care 33, 61-66.

[12] Scheltens P, Leys D, Barkhof F, Huglo D, Weinstein HC, Vermersch P, Kuiper M, Steinling M, Wolters EC, Valk J (1992) Atrophy of medial temporal lobes on MRI in "probable" Alzheimer's disease and normal ageing: Diagnostic value and neuropsychological correlates. J Neurol Neurosurg Psychiatry 55, 967-972.

[13] Warren MW, Hynan LS, Weiner MF (2012) Lipids and adipokines as risk factors for Alzheimer's disease. $J$ Alzheimers Dis 29, 151-157.

[14] Bigalke B, Schreitmuller B, Sopova K, Paul A, Stransky E, Gawaz M, Stellos K, Laske C (2011) Adipocytokines and CD34 progenitor cells in Alzheimer's disease. PLoS One 6, e20286.

[15] Johnston JM, Hu WT, Fardo DW, Greco SJ, Perry G, Montine TJ, Trojanowski JQ, Shaw LM, Ashford JW, Tezapsidis $\mathrm{N}$ (2014) Low plasma leptin in cognitively impaired ADNI subjects: Gender differences and diagnostic and therapeutic potential. Curr Alzheimer Res 11, 165-174.

[16] Khemka VK, Bagchi D, Bandyopadhyay K, Bir A, Chattopadhyay M, Biswas A, Basu D, Chakrabarti S (2014) Altered serum levels of adipokines and insulin in probable Alzheimer's disease. J Alzheimers Dis 41, 525-533.

[17] Reijmer YD, van den Berg E, Dekker JM, Nijpels G, Stehouwer CD, Kappelle LJ, Biessels GJ (2012) Development of vascular risk factors over 15 years in relation to cognition: The Hoorn Study. J Am Geriatr Soc 60, 1426-1433.

[18] Jack CR, Jr., Knopman DS, Jagust WJ, Petersen RC, Weiner MW, Aisen PS, Shaw LM, Vemuri P, Wiste HJ, Weigand SD, Lesnick TG, Pankratz VS, Donohue MC, Trojanowski JQ (2013) Tracking pathophysiological processes in Alzheimer's disease: An updated hypothetical model of dynamic biomarkers. Lancet Neurol 12, 207-216.

[19] Bonda DJ, Stone JG, Torres SL, Siedlak SL, Perry G, Kryscio R, Jicha G, Casadesus G, Smith MA, Zhu X, Lee HG (2014) Dysregulation of leptin signaling in Alzheimer disease: Evidence for neuronal leptin resistance. J Neurochem 128, 162-172. 